\title{
Effect of Polishing Procedure on the Roughness and Bacterial Adhesion of Provisional Restorative Materials
}

\section{Néstor Usiel Lara-Jara1, Gabriel Fernando Romo-Ramírez¹, María del Pilar Goldaracena-Azuara1, Antonio Aragón-Piña ${ }^{2}$, Claudia Butrón-Téllez Girón ${ }^{3 *}$, María Verónica Méndez-González ${ }^{1}$, Ana María González-Amaro ${ }^{1}$}

${ }^{1}$ Faculty of Stomatology, Autonomous University of San Luis Potosí, San Luis Potosí City, México

${ }^{2}$ Faculty of Engineering, Institute of Metallurgy, Autonomous University of San Luis Potosí, San Luis Potosí City, México ${ }^{3}$ Laboratory of Biochemistry, Microbiology and Pathology, Faculty of Stomatology, Autonomous University of San Luis Potosí, San Luis Potosí City, México

Email: anamara75@hotmail.com, *claudia.butron@uaslp.mx

How to cite this paper: Lara-Jara, N.U., Romo-Ramírez, G.F., Goldaracena-Azuara, M. del P., Aragón-Piña, A., Butrón-Téllez Girón, C., Méndez-González, M.V. and González-Amaro, A.M. (2018) Effect of Polishing Procedure on the Roughness and Bacterial Adhesion of Provisional Restorative Materials. Microscopy Research, 6, 9-18.

https://doi.org/10.4236/mr.2018.62002

Received: March 21, 2018

Accepted: April 24, 2018

Published: April 27, 2018

Copyright ( 92018 by authors and Scientific Research Publishing Inc. This work is licensed under the Creative Commons Attribution International License (CC BY 4.0).

http://creativecommons.org/licenses/by/4.0/

(c) (i) Open Access

\begin{abstract}
Provisional prosthetic restoration materials are exposed to oral cavity producing on the surface biofilm where different factors such as surface roughness and porosity can condition their formation and organization and can create stagnation areas that promote the bonding of organic particles, thus facilitating the formation and maturation of the biofilm. The purpose of this study was to compare surface roughness of two provisional prosthetic restorations materials and their bacterial susceptibility. In this study, two provisional restoration materials were used in two groups, A polymethyl methacrylate acrylic (NicTone MDC DENTAL) and B bis-acryl resin (Protemp 4 ESPE $3 \mathrm{M}$ ). A total of 80 samples (40 samples of each material) were in thick plates of $10 \times 10 \mathrm{~mm}$ and $2 \mathrm{~mm}$ high. 20 samples of each material were polished, while 20 were left unpolished. Subsequently, the samples were observed by Atomic Force Microscopy for their evaluation of surface roughness. The values were analyzed with t-test, Mann-Whitney $U$ test and Kruskal Wallis test. The samples were microbiologically inoculated with the strains obtained and identified from a provisional polymethyl methacrylate acrylic restoration in a patient, in order to observe bacterial adhesion using a Scanning Electron Microscope. Two strains, Enterococcus faecalis and Pseudomona luteola, were identified. The presence of the microorganisms was observed on the surface of both materials, either polished or unpolished, with a lower level of microorganism adhesion found on the bis-acrylic resin. There was a significant difference about surface roughness in the groups A and B with $\mathrm{p}<0.05$; mean-
\end{abstract}


while there was also a significant difference between polished polymethyl methacrylate and polished bis-acrylic resin $\left(\mathrm{p}=6.7 \times 10^{-8}\right)$. We found that the polished bis-acrylic resin showed lower surface roughness and bacterial adhesion in comparison with the polymethyl methacrylate.

\section{Keywords}

Surface Analysis, Bacterial Adhesion, Provisional Fixed Restoration

\section{Introduction}

Treatment with fixed prosthesis requires the prepared teeth to be protected and stabilized with a provisional restoration that resembles the form and function of the definitive treatment planned [1].

Multiple areas of particular concern with the use of provisional restorations have been identified, including aesthetic, comfort, phonetic and functional issues, as well as ones related to maxilomandibular areas and periodontal health [1].

The materials available for the production of provisional prostheses include polymethyl methacrylate, polymethyl methacrylate and bis-acrylic resin [2], as well as polyvinyl methacrylate, urethane methacrylate and microfill resin [3]. The principal requirements for provisional materials are as follows: an adequate marginal adaptation; fracture resistance; low thermal conductivity; a non-irritant reaction with dental pulp and gingival tissue; and, ease of cleaning [2]. The majority of the bacteria in the oral cavity can only survive if they adhere to its various hard surfaces (the tooth itself, filling material, dental implants or prosthesis), all of which have different surface characteristics [4].

The fixings of certain microorganisms to specific surfaces in the oral cavity and the formation of dental plaque on either the tooth or dental materials are the primary causes of oral diseases such as denture stomatitis, gingival inflammation, and secondary cavities [5] [6], all of which can lead to serious health complications [5].

For this reason, bacteria are of considerable importance in the early formation of plaque, not only on the natural tissue of the teeth, but also on modern resin-based restorative dental materials [7]. Thus, the surface of the provisional crown or fixed prosthesis must be sufficiently soft in order to be comfortable, aesthetically pleasing and capable of avoiding both stains and the accumulation of plaque [5]. For this reason, the appropriate finishing and polishing of dental materials are important aspects in the procedures involved in clinical restoration [6].

Considering that bacterial adhesion on hard dental surfaces is followed by the accumulation of dental plaque, in which process surface roughness and free surface energy play a key role [4] [8], changes in these clinically important variables can have a significant influence on bacterial adhesion and retention [7]. 
It has been shown that rough acrylic resin surfaces are significantly more prone to the accumulation of bacteria and the formation of plaque than soft surfaces [9]. Thus, the objective of this study was to compare the surface roughness of two fixed provisional prosthodontic materials (polymethyl methacrylate and bis-acrylic resin), based on their susceptibility to bacterial adhesion of microorganisms obtained from the provisional crowns of a patient through the use of the Scanning Electron Microscope (SEM) and Atomic Force Microscopy (AFM).

\section{Materials and Methods}

An in vitro experimental study approved by the Ethics in Research Committee of the Faculty of Stomatology at the UASLP, with the code CEI-FE-058-015, while the hazardous biological infectious waste was treated in accordance with Official Mexican Standard 087-ECOL-SSA1-2012.

\subsection{Preparation of the Samples}

A total of 80 samples were divided in two groups of materials (40 samples of each material), group A used polymethyl methacrylate acrylic (NicTone MDC DENTAL) and group B used bis-acrylic resin (Protemp 4 ESPE 3M). Each group was random divided into two subgroups: polished and unpolished. All samples were fabricate in thick plates of $10 \times 10 \mathrm{~mm}$ and $2 \mathrm{~mm}$ high using an A-silicon guide (Variotime HERAEUS) and handling the material in accordance with the manufacturer's specifications. Each material was mixed and polymerized according to the manufacturer's instructions. From both groups 20 samples were polished with polishing wheels and acrylic polishing paste (polycril MDC DENTAL), while 20 were left unpolished.

One operator polished all the samples, was blinded to the material that was being polished. The samples were all polished in the same orientation, and all were observed for surface roughness along the same orientation. After polishing, each sample was rinsed under distilled water and dried on plates over absorbent paper.

\subsection{Atomic Force Microscopy (AFM) Analysis}

All samples were evaluated at the same scan size $\left(1 \times 1 \mu \mathrm{m}^{2}\right)$, by triplicate in different areas, of all selected the mean roughness obtained for each sample were used for the statistical analysis. The evaluations of the samples surface roughness were carried out using an AFM (Jeol JSM-6610LV; Jeol Ltd, Akishima, Tokyo, Japan) in the tapping mode. The Nanoscope 5.31r1 software was used to measure the AFM parameters.

A descriptive statistics was performed for the surface roughness of all subgroups. T-test and Mann-Whitney U tests were used to compare the difference within groups. Kruskal Wallis and the Bonferroni test for post-hoc to compare the subgroups. R program version 3.5.0 was used for statistical analysis. Statistical significance was set at $\mathrm{p}<0.05$. 


\subsection{Obtaining the Microorganisms}

Samples were taken from a fixed provisional polymethyl methacrylate-based prosthesis in a patient for the cultivation and identification of the microorganisms.

The fixed provisional prosthesis was removed from the patient under relative isolation, softly brushed and washed with sterile distilled water, from which a portion was cut from the provisional prosthesis with a low speed handpiece with a diamond disc burr. The sample obtained was placed in an enriched culture medium (trypticasein soy broth) and incubated for 24 hours in order to obtain a mixed culture (primary culture). The sample was then emptied onto a blood agar plate (Becton Dickinson and Company, Mexico S.A. de C.V.) for the macroscopic differentiation (UFC) (microscope-LEICA EZ4 D, Leica Microsystems $\mathrm{GmBH}$, Wetzlar, Germany) and microscopic differentiation of the colonies (gram stain) (microscope-LEICA DM500, Leica Microsystems GmBH, Wetzlar, Germany). Once the colonies had been differentiated, a pure culture was then taken in order to identify them via API 20E enzymatic tests (Analytical Profile Index, Biomeriéux, France) and API 20 STREP (Analytical Profile Index, Biomeriéux, France).

\subsection{Innoculation of the Samples}

The microbiological inoculation of the samples with the strains identified was carried out by placing them in a liquid culture medium of trypticase in soy broth and incubating them for 48 hours at $35^{\circ} \mathrm{C} \pm 2^{\circ} \mathrm{C}$, with the trypticase in soy broth liquid culture medium replaced every $24 \mathrm{hrs}$ for 15 days in order to facilitate posterior observation in the SEM.

\subsection{Preparation of the Samples for Observation in the SEM}

All samples were first washed in $0.1 \mathrm{M}$ phosphate-buffered saline solution, fixed with $2 \%$ glutaraldehyde (Sigma-Aldrich, St. Louis, MO, USA) and $1 \%$ Alcian Blue Stain 8GX (Sigma Aldrich, St. Louis, MO, USA), and then stored at $4^{\circ} \mathrm{C}$ for 24 hours. Once fixed, the samples were washed three times with $0.1 \mathrm{M}$ phosphate-buffered solution in order to eliminate excess material. The samples were dehydrated in a pure anhydrous ethanol series (Industrial Chemical Technology Ltd.) of $20 \%, 40 \%, 60 \%, 80 \%, 90 \%$ and $95 \%$ for 10 minutes in each series, and then finally submerged in pure ethanol, with the critical point achieved in a critical point dryer (CPD 030 BAL-TEC GmbH, Schalksmühle, Germany). The samples were coated via sputtering with a $20 \mathrm{~nm}$ gold-palladium mix (Fine Layer of Sputter Ions JFC-1100, EE.UU.). The samples were evaluated with the SEM (JEOL JSM-6610 LV, Japan) at different magnifications and a low kilovoltage (5 Kv).

\section{Results}

Characteristics of the surface roughness ( $\mathrm{Ra}$ ) values of all materials were performed for each subgroup (Table 1). There were statistically significant differ- 
ences in the group A and B with $\mathrm{p}=6.7 \times 10^{-8}$ and $\mathrm{p}=2.2 \times 10^{-16}$ respectively.

Statistically significant differences were observed among surface roughness (Ra) in subgroups $(\mathrm{p}<0.05)$ except when comparing the polished polymethyl methacrylate subgroup with the polished bis-acrylic resin subgroup $(\mathrm{p}>0.05)$ (Table 2).

However a significant difference was observed when comparing the two subgroups of polished materials $\mathrm{p}=6.7 \times 10^{-8}$.

The results for the samples taken from the patients in accordance with the macroscopically and microscopically identified colonies revealed two types, identified as Enterococcus faecalis and Pseudomona luteola with the apiweb software, obtaining the percentages shown in Table 3.

The images obtained in the SEM from group A (polymethyl methacrylate) and group B (bis-acrylic resin) polished and unpolished showed microorganism

Table 1. Characteristics of surface roughness $(\mathrm{Ra})$ of the subgroups.

\begin{tabular}{ccc}
\hline & Mean \pm SD & Median IQR \\
\hline Group A & \\
& & 22.7 \\
Unpolished polymethyl methacrylate & $23.2 \pm 3.7$ & 3.8 \\
& & 9.34 \\
Polished polymethyl methacrylate & $9.4 \pm 0.7$ & 1.08 \\
Group B & & \\
& & 35.9 \\
Unpolished bis-acrylic resin & $36.6 \pm 8.2$ & 11.4 \\
& & 6.9 \\
Polished bis-acrylic resin & $6.7 \pm 0.5$ & 0.6 \\
\hline
\end{tabular}

${ }^{\star}$ Non-normal distribution.

Table 2. Statistical analysis (p values) of surface roughness ( $\mathrm{Ra}$ ) values; Kruskal Wallis and Bonferroni test for post-hoc.

\begin{tabular}{cccc}
\hline & $\begin{array}{c}\text { Unpolished polymethyl } \\
\text { methacrylate }\end{array}$ & $\begin{array}{c}\text { Polished polymethyl } \\
\text { methacrylate }\end{array}$ & $\begin{array}{c}\text { Unpolished } \\
\text { bis-acrylic resin }\end{array}$ \\
\hline $\begin{array}{c}\text { Polished polymethyl } \\
\text { methacrylate }\end{array}$ & $2.5 \times 10^{-14}$ & - & - \\
Unpolished bis-acrylic resin & $1.2 \times 10^{-13}$ & $\mathrm{p}<0.001$ & - \\
$\begin{array}{c}\text { Polished bis-acrylic resin } \\
\text { m }\end{array}$ & $\mathrm{p}<0.001$ & 0.39 & $\mathrm{p}<0.001$ \\
\hline
\end{tabular}

Table 3. Microorganisms identified of samples taken from provisional fixed prostheses of a patient.

\begin{tabular}{cccc}
\hline Colony & Gram & Name & Porcentaje of Identification \\
\hline 1 & Cocos Gram+ & Enterococcus faecalis & 99.7 \\
2 & Bacilos Gram- & Pseudomona luteola & 99.9 \\
\hline
\end{tabular}


adherence in an analog colonization pattern on all the surfaces of the materials observed and a variable number of microorganism adhesions.

The image (Figure 1(a)) presents the topography of the unpolished bis-acrylic resin, showing rough areas and, on the edges, grooves and cracks which facilitate the colonization, as indicated with arrows. An enlargement of the area (Figure $1(b)$ ), shows metabolically active bacterial settlements in this area, with imperfections on the surface. In Figure 1(c) and Figure 1(d), a polished bis-acrylic resin is observed, with a more regular surface on which some microorganisms are distributed.

Figure 2(a) Presents a surface less rough in polished polymethyl methacrylate in comparison with unpolished bis-acrylic resin, although with presence of defects and pores as well as microorganisms in the surface as indicated in the highlighted areas, with the magnification of $2000 \times$. Figure $2(b)$ indicates same area at a $4000 \times$ magnification showing microorganisms in groups as well as isolated appreciating in a better way the imperfections of the surface. Figure $2(\mathrm{c})$ and Figure 2(d) polymethyl methacrylate polished sample at $2000 \times$ and $4000 \times$, respectively, in which a regular surface can be seen with the presence of pores and scratches, as well as the presence of microorganisms across the surface, as indicated in the highlighted areas.

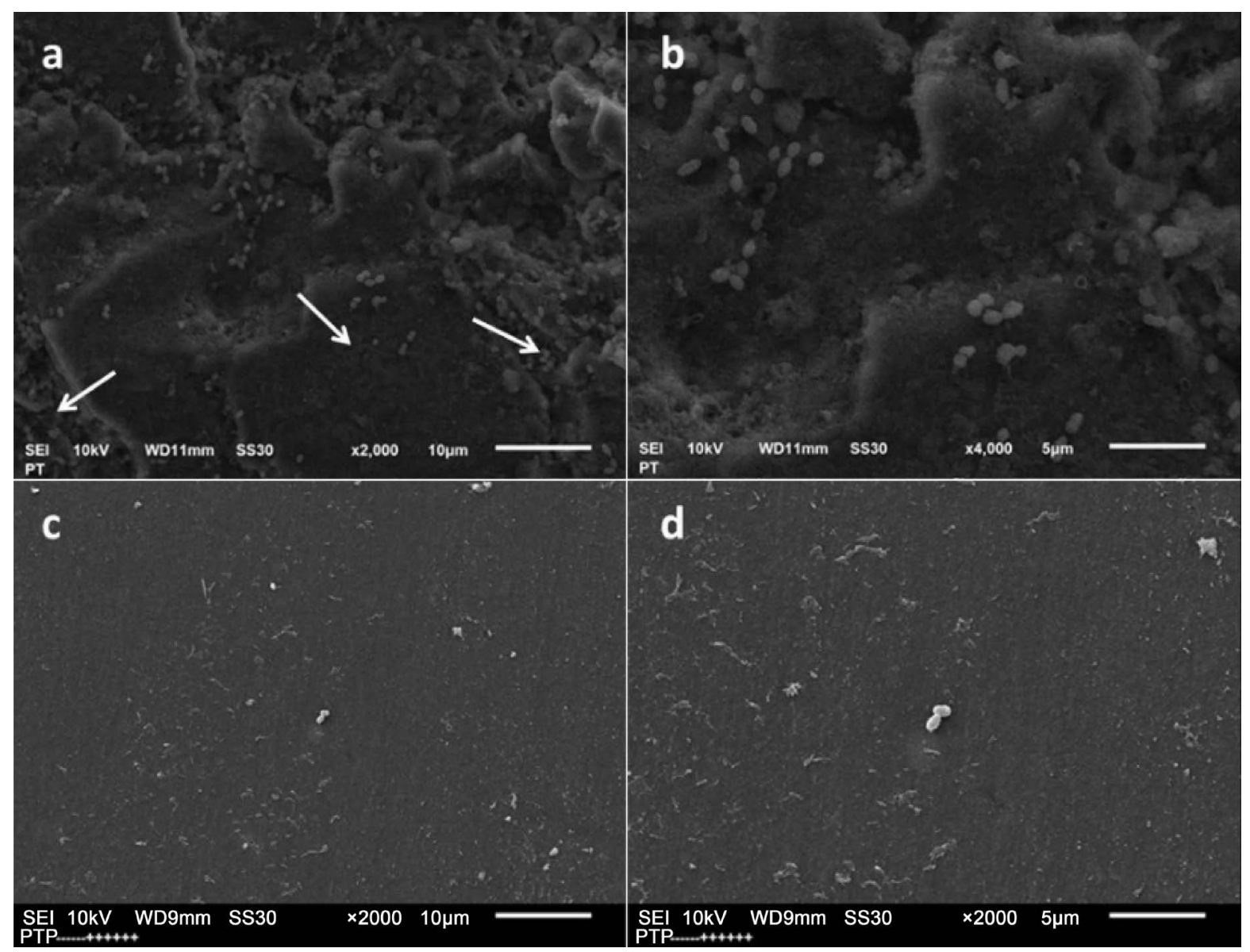

Figure 1. Images of unpolished ((a), (b)) and polished ((c), (d)) bis-acrylic resin at magnifications of 2000× and 4000×. 


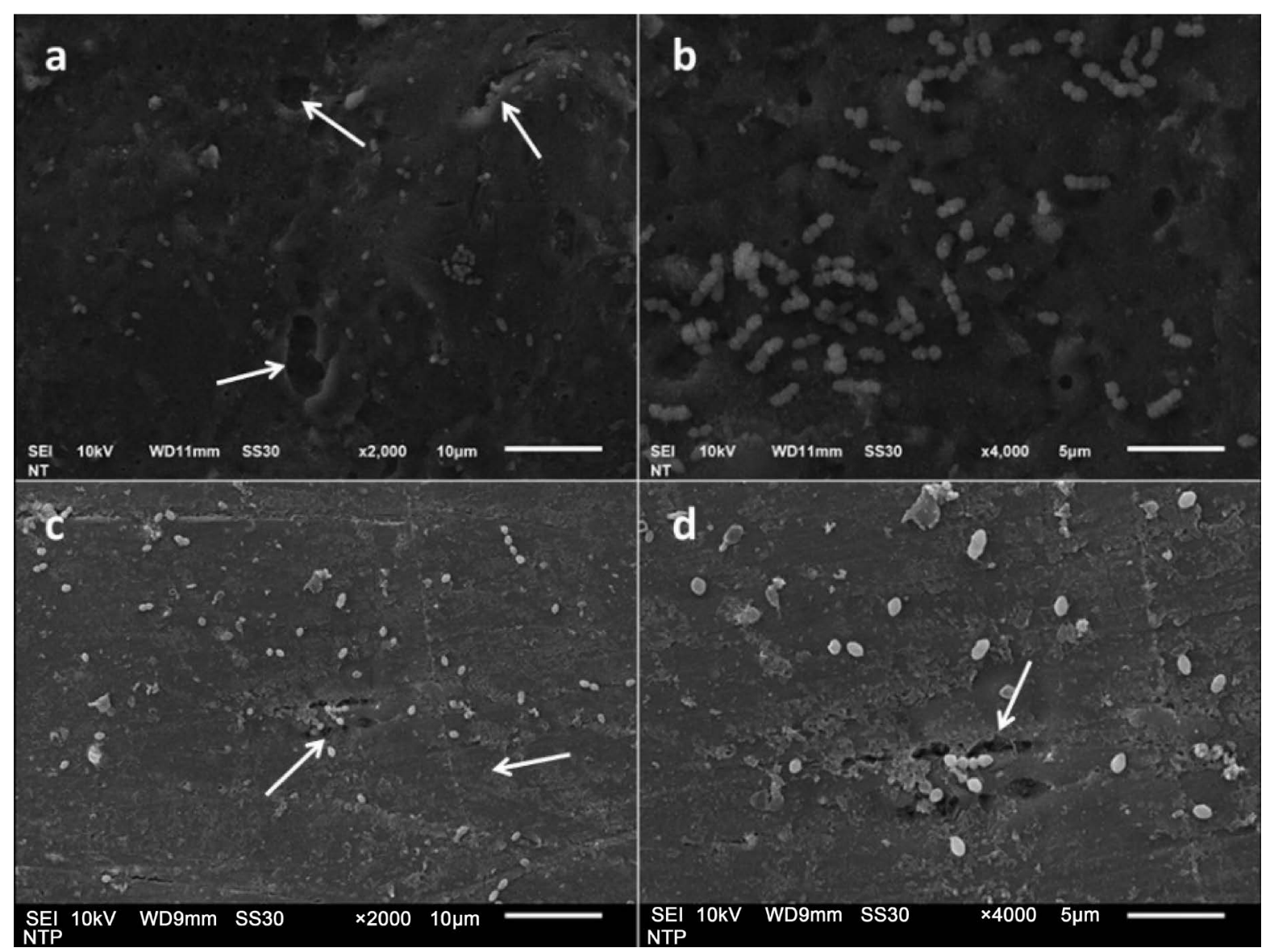

Figure 2. Presence of defects as well as microorganisms across the entire surface in groups and isolated.

\section{Discussion}

The differences in the physico-chemical characteristics are the reason that some materials are more prone to bacterial adhesion and the formation of plaque than others. Rough surfaces and free surface energy are the two most significant determinants of bacterial adhesion [10].

The oral cavity is constantly contaminated by a complex diversity of microbial species with a strong tendency to colonize surfaces [11] [12], with the adhesion of said organisms to the rough surfaces of the mouth facilitating their survival [13] [14]. The primary colonizing microorganisms of the surfaces, which are causes of oral diseases, are from the Streptococcus mutans and Streptococcus sobrinus group [15].

Both Buergers et al. [5] and Montanaro et al. [16] reported in vitro studies that quantify adhesion to various materials. These studies used these microorganisms with commercial strains, specifically Streptococcus mutans, to inoculate provisional restoration materials such as bis-acrylic resins, polymethyl methacrylate and direct restoration materials, using techniques to determine their roughness and an SEM to observe the presence of colonization on their surface.

This study used strains from a polymethyl methacrylate-based provisional restoration thus eliminating the presence of enamel on the surface removed 
from a patient completely rehabilitated with a fixed prosthesis. The strains identified correspond to Enterococcus faecalis and Pseudomona luteola and are different to those used in other studies. The main reason why the study used the strains identified from a provisional restoration sample stems from the fact that the substrates used for contamination are of a synthetic and non-organic origin, unlike dental enamel, with the adhesins of the microorganisms specific to each substrate. Cariogenic microorganisms use their affinity with enamel in order to be able to reproduce. It should also be noted that the microorganisms identified as present in the provisional restoration are opportunistic pathogens that cause nosocomial infection.

This is the case with Enterococcus faecalis, which is very difficult to eliminate when found in deep tissue and is responsible for $80 \%$ of human infection by enterococci. It is an extremely resistant organism that is well adapted for survival and has good development, even under certain conditions that would be lethal for many related microorganisms [17].

Buergers et al. [5] reported that the quantity of bacterial adhesion was significantly different among the provisional materials studied and that it was not possible to confirm a correlation between bacterial adhesion and surface roughness. Furthermore, bis-acrylic resin and polymethyl methacrylates were shown to have significantly lower adhesion potential than most methacrylates.

The results in our study indicate the lower potential of bacterial adhesion in the group of bis-acrylic resin in comparison with the polymethyl methacrylate group. However, evaluating the surface roughness of the subgroups, the unpolished bis-acrylic resin was rougher than the unpolished polymethyl methacrylate although in this last one was observed that its high potential bacterial adhesion had no influence in its roughness whereby the variability in microorganisms colonization could depend of the substrate.

Knowledge as to how the surface of dental materials affects the accumulation and proliferation of a number of species of microorganisms is useful, as it would provide information on how to take a decision on the alternatives for the manufacture of a provisional restoration. It will also provide a perspective on the interaction between these materials and microorganisms during their lifespan in the oral cavity, as the rough surface, to a greater or lesser degree, will facilitate the accumulation of microorganisms and particles.

\section{Conclusions}

The microorganisms identified from a polymethyl methacrylate acrylic-based restoration are not the same as the primary colonizers found on dental surfaces, due to the difference in their affinity with the substrate.

Within the limitations of this study, the following conclusions were drawn:

1) Surface roughness was different according to the type of prosthetic provisional restoration materials and the polishing procedure.

2) The samples of polymethyl methacrylate group showed a greater bacterial 
adhesion in comparison with the group of bis-acrylic resin.

3) Increased surface roughness was not directly related to increment bacterial adhesion the samples unpolished of the bis-acrylic resin.

4) Increased bacterial adhesion was directly related to the decrease of the surface roughness unpolished polymethyl methacrylate.

5) Polishing of the samples presented smoother surfaces reducing the surface roughness and the bacterial adhesion finding the polished bis-acrylic resin with the lowest bacterial adhesion and surface roughness.

\section{Acknowledgements}

This study was supported by the Fondo de Apoyo a la Investigación (FAI) of the Universidad Autónoma de San Luis Potosí.

\section{Conflict of Interest}

The authors declare they have not conflict of interests.

\section{References}

[1] Burns, D.R, Beck, D.A. and Nelson, S.K. (2003) A Review of Selected Dental Literature on Contemporary Provisional Fixed Prosthodontic Treatment: Report of the Committee on Research in Fixed Prosthodontics of the Academy of Fixed Prosthodontics. The Journal of Prosthetic Dentistry, 90, 474-497.

https://doi.org/10.1016/S0022-3913(03)00259-2

[2] Ayuso-Montero, R., Martinez-Gomis, J., Lujan-Climent, M., Salsench, J. and Peraire, M. (2009) Influence of Matrix Type on Surface Roughness of Three Resins for Provisional Crowns and Fixed Partial Dentures. Journal of Prosthodontics, 18, 141-144. https://doi.org/10.1111/j.1532-849X.2008.00392.x

[3] Guler, A.U., Kurt, S. and Kulunk T. (2005) Effects of Various Finishing Procedures on the Staining of Provisional Restorative Materials. The Journal of Prosthetic Dentistry, 93, 453-458. https://doi.org/10.1016/j.prosdent.2005.02.001

[4] Sen, D., Göller, G. and Issever, H. (2002) The Effect of Two Polishing Pastes on the Surface Roughness of Bis-Acryl Composite and Methacrylate-Based Resins. The Journal of Prosthetic Dentistry, 88, 527-532. https://doi.org/10.1067/mpr.2002.129335

[5] Buergers, R., Rosentritt, M. and Handel, G. (2007) Bacterial Adhesion of Streptococcus mutans to Provisional Fixed Prosthodontics Material. The Journal of Prosthetic Dentistry, 98, 461-469. https://doi.org/10.1016/S0022-3913(07)60146-2

[6] Kuhar, M. and Funduk, N. (2005) Effects of Polishing Techniques on the Surface Roughness of Acrylic Denture Base Resins. The Journal of Prosthetic Dentistry, 93, 76-85. https://doi.org/10.1016/j.prosdent.2004.10.002

[7] Rüttermann, S., Bergmann, N., Beikler, T., Raab, W.H. and Janda, R. (2012) Bacterial Viability on Surface-Modified Resin-Based Dental Restorative Materials. Archives of Oral Biology, 57, 1512-1521.

https://doi.org/10.1016/j.archoralbio.2012.05.005

[8] Bollen, C.M., Lambrechts, P. and Quirynen, M. (1997) Comparison of Surface Roughness of Oral Hard Materials to the Threshold Surface Roughness for Bacterial Plaque Retention: A Review of the Literature. Dental Materials, 13, 258-269. 
https://doi.org/10.1016/S0109-5641(97)80038-3

[9] Morgan, T.D. and Wilson, M. (2001) The Effects of Surface Roughness and Type of Denture Acrylic on Biofilm Formation by Streptococcus oralis in Constant Depth Film Fermentor. Journal Applied Microbiology, 91, 47-53. https://doi.org/10.1046/j.1365-2672.2001.01338.x

[10] Sardin, S., Morrier, J.J., Benay, G. and Barsotti, O. (2004) In Vitro Streptococcal Adherence on Prosthetic and Implant Materials. Interactions with Physicochemical Surface Properties. Journal of Oral Rehabilitation, 31, 140-148. https://doi.org/10.1046/j.0305-182X.2003.01136.x

[11] Gharechahi, M., Moosavi, H. and Forghani, M. (2012) Effect of Surface Roughness and Materials Composition on Biofilm Formation. Journal of Biomaterials and Nanobiotechnology, 3, 541-546. https://doi.org/10.4236/jbnb.2012.324056

[12] Busscher, H.J., Rinastiti, M., Siswomihardjo, W. and van der Mei, H.C. (2010) Biofilm Formation on Dental Restorative and Implant Materials. Journal of Dental Research, 89, 657-665. https://doi.org/10.1177/0022034510368644

[13] Quirynen, M. and Bollen, C.M. (1995) The Influence of Surface Roughness and Surface-Free Energy on Supra- and Subgingival Plaque Formation in Man. A Review of the Literature. Journal of Clinical Periodontology, 22, 1-14.

https://doi.org/10.1111/j.1600-051X.1995.tb01765.x

[14] Quirynen, M., Marechal, M., Busscher, H.J., Weerkamp, A.H., Darius, P.L. and van Steenberghe, D. (1990) The Influence of Surface Free Energy and Surface Roughness on Early Plaque Formation. An in Vivo Study in Man. Journal of Clinical Periodontology, 17, 138-144. https://doi.org/10.1111/j.1600-051X.1990.tb01077.x

[15] Liljemark, W.F. and Bloomquist, C. (1996) Human Oral Microbial Ecology and Dental Caries and Periodontal Diseases. Critical Reviews in Oral Biology and Medicine, 7, 180-198. https://doi.org/10.1177/10454411960070020601

[16] Montanaro, L., Campoccia, D., Rizzi, S., Donati, M.E., Breschi, L., Prati, C. and Arciola, C.R. (2004) Evaluation of Bacterial Adhesion of Streptococcus mutans on Dental Restaurative Materials. Biomaterials, 25, 4457-4463.

https://doi.org/10.1016/j.biomaterials.2003.11.031

[17] Sundqvist, G., Figdor, D., Persson, S. and Sjögren, U. (1998) Microbiologic Analysis of Teeth with Failed Endodontic Treatment and the Outcome of Conservative Re-Treatment. Oral Surgery, Oral Medicine, Oral Pathology, Oral Radiology, and Endodontology, 85, 86-93. https://doi.org/10.1016/S1079-2104(98)90404-8 